\title{
Improving Focus and Context Awareness in Interactive Visualization of Time Lines
}

\author{
Saturnino Luz \\ Department of Computer Science \\ Trinity College Dublin, Dublin, Ireland \\ luzs@cs.tcd.ie
}

\author{
Masood Masoodian \\ Department of Computer Science \\ The University of Waikato, Hamilton, New Zealand \\ masood@cs.waikato.ac.nz
}

\begin{abstract}
This paper presents an analysis and empirical evaluation of techniques developed to support focus and context awareness in tasks involving visualization of time lines. It focuses on time lines that display discrete events and their temporal relationships. The most common form of representation for such time lines is the Gantt chart. Although ubiquitous in event visualization and project planning applications, Gantt charts are inherently space-consuming, and suffer from shortcomings in providing focus and context awareness when a large number of tasks and events needs to be displayed. In an attempt to address this problem, we implemented and adapted a number of focus and context awareness techniques for an interactive task scheduling system in combination with the standard Gantt chart and an alternative space-filling mosaic approach to time line visualization. A controlled user trial compared user performance at interpreting representations of hierarchical task scheduling, assessing different methods across various conditions resulting from interactive explorations of the Gantt and the mosaic interfaces. Results suggested a number of possible improvements to these interactive visualization techniques. The implementation of some of these improvements is also presented and discussed.
\end{abstract}

Time lines, Gantt charts, temporal mosaic, information visualization, evaluation, task hierarchies, focus and context awareness.

\section{INTRODUCTION}

Time lines provide a flexible and intuitive form of representation of events over time. According to the taxonomy proposed in [1], while events are often represented through multiple numeric variables at different levels of abstraction following a linear structure (as in [14, 11], for instance), perhaps the most common use of time lines is in representing univariate data in the form of time intervals. The latter is a form widely used in project planning and scheduling visualizations such as the popular Gantt chart, and its modern interactive variants such as LifeLines [21], PlanningLines[2] etc. In such charts, the data are simply encoded as labels for activities scheduled over time intervals stretching across different levels of a branching time structure.

While Gantt-style charts, which display activities or events as parallel (typically horizontal) bars along the time axis, are very common, alternative designs have been proposed. A space-filling time line, or "temporal mosaic", which allocates space proportionally to the number of activities or events scheduled for a time interval was presented in [17]. In an evaluation of the alternative time lines as static visualizations in a multimedia browsing scenario, users of that spacefilling time line performed as well as or better than users of its traditional Gantt-style counterpart.

As Tufte observed [25], when rendered as static graphics for project planning and scheduling, time lines take up a lot of space. On small computer screens, however, time lines gain an interactive dimension which can help mitigate this problem. The most obvious way of managing limited screen space is by providing for scrolling and panning. While, these techniques are straightforward to implement, they can cause the user to become disoriented and unable to visualize high level relationships among tasks and sub-tasks when presented with a reasonably complex schedule. Over the years, a number of general techniques have been developed to preserve focus and context awareness in face of screen space limitations, including overview+detail layouts, zooming, blurring of non-focal items and various distortion-based techniques [23, 16, 15]. A few systems that deal with time-oriented data have employed these techniques. Somewhat surprisingly, however, there are very few studies specifically on the use of such techniques in combination with time lines.

This paper presents a study of the effects of several techniques designed to help the user maintain focus and context awareness while interacting with time lines representing task hierarchies. The experiment is based on an interactive time line visualization system which implements both a standard Gantt-like display and the aforementioned space-filling visualization, as well as several mechanisms for focus and context awareness. The effects of overview+detail through "collapsing" and "expanding" of task groups, distortion though a bifocal display technique, and "blurring" of non-focal items are analysed in 
combination with the two styles of time lines. To the best of our knowledge, this is the first study of the use of these techniques in connection with hierarchical task visualization both in its traditional form and its space-filling variant. Discussions of the shortcomings of each approach are also presented, along with a range of improvements we have now made to our prototype system in order to overcome these shortcomings.

\section{FOCUS AND CONTEXT AWARENESS}

Although time lines were originally conceived as a form of static visualization [26], a recent survey of their applications [22] attests to the fact that this static metaphor also carries through to interactive contexts. It has been used, for instance, in interactive systems for visualizing personal histories [21], displaying travel itinerary information across time-zones on conventional computer displays [5] or mobile devices [20], browsing multimedia collections [13], and many other tasks.

The techniques employed by such systems for supporting focus and context awareness vary a great deal. In [20], the alignment of time-zone adjusted calendar lines is employed to show associations between individual travel events across time. The LifeLines system [21] provides zooming, tied to different levels of time granularity, filtering and support for grouping of events through "facets" which can be opened or closed to show different aspects of the same information structures. Both faceted browsing and hierarchical organisation have been used in exploratory search applications [3] which have gained some popularity on the Web in recent years. These include MIT's SIMILE time line [12] and the Continuum time line visualization tool [4]. Both SIMILE and Continuum provide an overview+detail layout option, interactive zooming and filtering as well as detail on selected events ("brushing").

A different approach to handling context and focus is the Perspective Wall [19], which renders the time line as a 3D object whose centre shows a detailed view of events (focus), while the sides give the context in simulated perspective. A flexible widget specifically designed for zooming which supports distortions, including perspective and fish-eye view is presented in [9] which improves on some of the techniques introduced in the DataLens system [6].

Visualization of hierarchical information has been dealt with comprehensively by the TimeTree [8] and Timeline Trees [7] systems. TimeTree was designed to support browsing of large hierarchies displayed over a time axis, and therefore the system had to implement mechanisms for filtering, zooming etc. One of the mechanisms employed to deal with large datasets is the "folding" of sub-trees according to a degree-of-interest function [10]. Similar mechanisms are available in the Timeline Trees system [7], in which different time lines are laid out in front of the tree nodes rather than in parallel to the entire hierarchy, as in [8]. It is interesting to note that, like our system (described in the next section) Timeline Trees also employs a space-filling approach to the display of temporal information. Its use of space-filling visual elements, however, is limited to thumbnails (which present a sort of overview for the time line associated with a node in the hierarchy), and to an encoding similar to stacked bar charts for different event classes on the expanded time lines. Our system, on the other hand, encodes the hierarchy itself as a space-filling chart.

\section{AN INTERACTIVE TIMELINE SYSTEM}

An interactive prototype system was developed to allow better investigation of the effiectiveness of a range of focus and context awareness techniques, for both Gantt and the alternative space-filling (temporal mosaic) visualizations.Since an earlier version of this system has been more fully described elsewhere [18], here we limit ourselves to providing an overview of the features that are relevant to the study described and the issues discussed in this paper. These features are: expansion and collapsing of task representations, blurring out of details that are not in the user's focus of attention, zooming and distortion (bifocal display).

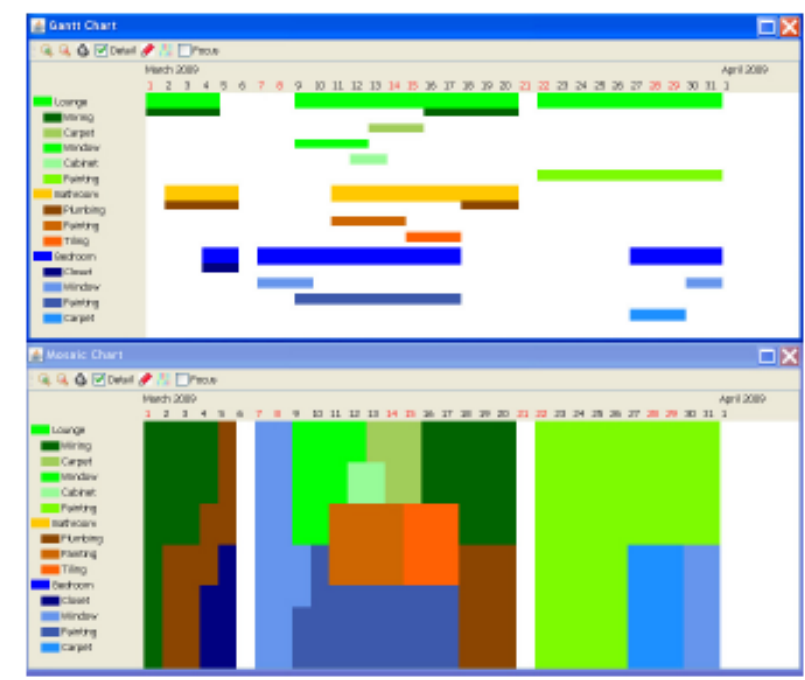

Figure 1: Interactive prototype system, showing the Gantt (top) and mosaic (bottom) visualizations.

Figure 1 shows an earlier version of the interface of our interactive visualization prototype. The top window shows aschedule for a house renovation project in Gantt chart, and the bottom window shows the same schedule in mosaic visualization. As can be seen in this figure, while the Gantt chart reserves space and uses parallel time lines to display duration of individual tasks or sub-tasks when they are scheduled to take place, the mosaic visualization 
uses the same area of space to proportionally show only the tasks or sub-tasks that are scheduled for a particular time period.

The simplest form of focus and context awareness feature that our system provides, is to allow abstraction of sub-task details of higher level tasks. Figure 2 shows how sub-tasks of all tasks can be collapsed (or "folded away") and hidden in both Gantt and mosaic visualizations. It is also possible to expand the sub-tasks of one or more tasks, while still keeping the other tasks collapsed. Notice that the overall shape and amount of screen space occupied by the mosaic does not change as the tasks are collapsed and expanded, while the Gantt chart grows or contracts along the vertical axis when tasks are expanded or collapsed. The use of these features might have implications with respect to context awareness.

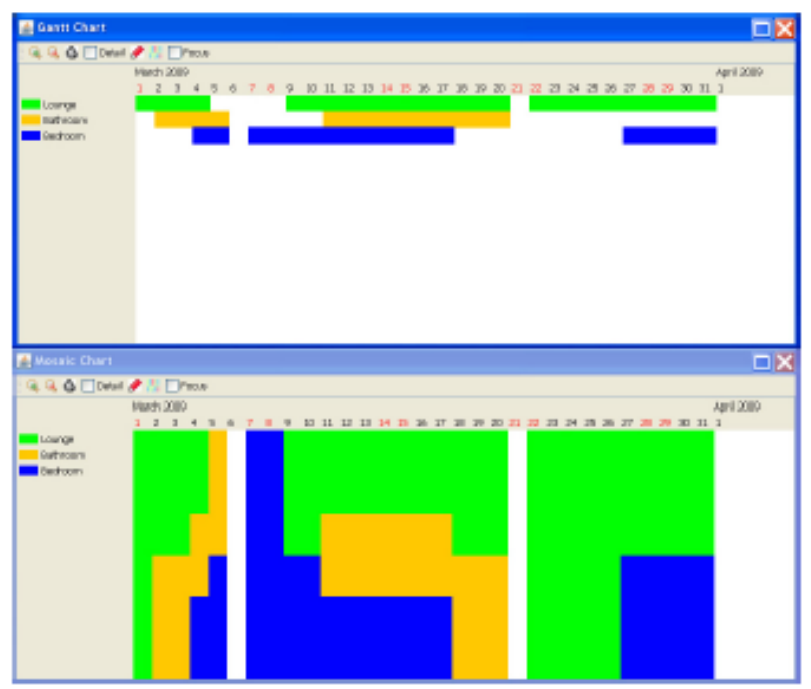

Figure 2: Gantt and mosaic visualizations with sub-tasks folded away.

If, on the one hand, shape changes (on Gantt charts) provide the user with conspicuous indication that a transition of system state is taking place, those shape changes may, on the other hand, prove disruptive in that the user may need to visually rescan the whole chart in order to visualize an overview of the schedule.

Another feature of the system is its mechanism for blurring out details of some tasks and their subtasks, while allowing the viewing of a user-specified set of specific tasks or sub-tasks in detail. Figure 3 shows a chart in which the details of all the tasks and their sub-tasks have been blurred, except for the focal Lounge task and its sub-tasks which are fully visible (i.e. in "sharp" view). It is also possible to blur out all tasks and their sub-tasks, while only showing a group of tasks or sub-tasks that share a common attribute. In Figure 4, for instance, all the sub-tasks are blurred while only painting sub-tasks across all three main tasks are fully visible.

In addition to the above mentioned features, we have implemented a form of zooming based on the bifocal display technique [16], which we also aimed to assess. The bifocal display allows the user to see more details of all the tasks or sub-tasks within a specific time period by zooming into sections of the time line using a tool similar to a magnifying glass. However, unlike a magnifying glass, this type of zoom tool preserves the continuity of tasks and subtasks when moving from the inside of the zoomed area to the outside. Figure 5 shows the zoom function applied to days 10 to 14 , where details of sub-tasks, including their names, are displayed. The zoom function allows the user to set the time span, as well as the zoom level of the magnification, applied to the selected area.

\section{EVALUATION}

Previous experiments have shown the effectiveness of the static (non-interactive) temporal mosaics against conventional time lines (including Gantt charts) for a number of visualization tasks [17]. The study presented below, in contrast, aims to evaluate features which are relevant to the use

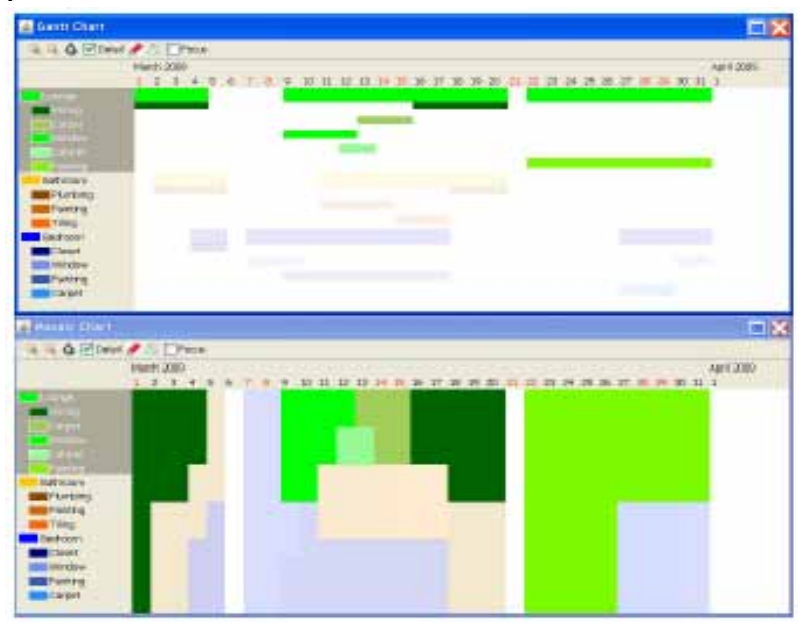

Figure 3: Gantt and mosaic visualizations with most subtasksblurred.

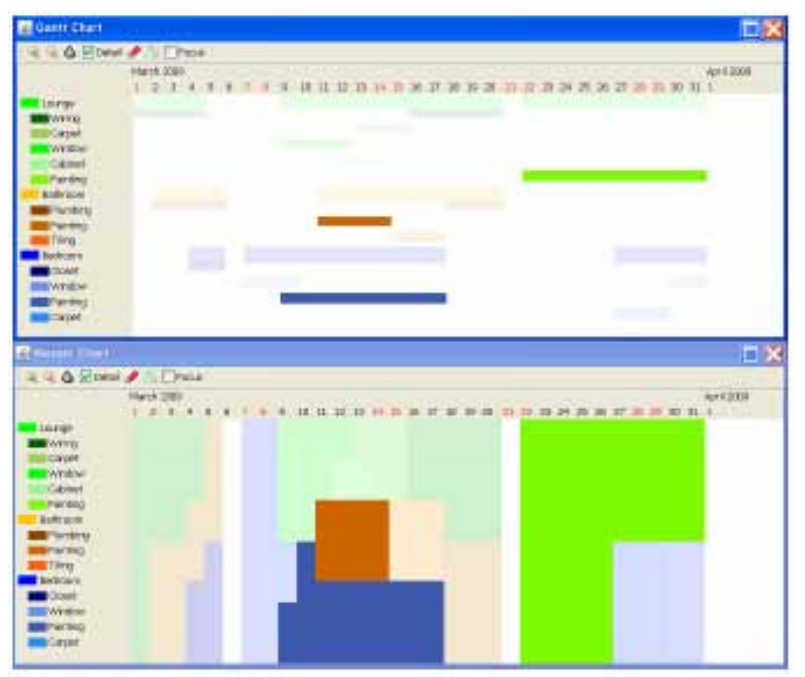

Figure 4: Gantt and mosaic visualizations with all but painting sub-tasks blurred. 
of these basic visualizations in interactive contexts. In particular, we assess how a range of focus and context awareness mechanisms, as implemented in our interactive prototype system, might affect user performance when used in conjunction with temporal mosaic and Gantt charts to analyse a project schedule.

It should be noted, however, that we decided to compare the Gantt and mosaic visualizations using static images generated through our interactive mechanisms, rather than allowing the study participants to use the interactive prototype itself. We decided on this setup in order to make sure that for each study task the two visualizations were compared using exactly the same area of the schedule, viewing area size, etc. Therefore, the study was not about the usability of the interactive system, but rather aimed to compare the visualizations generated using the focus and context mechanisms in mosaic and Ganttstyle time lines.

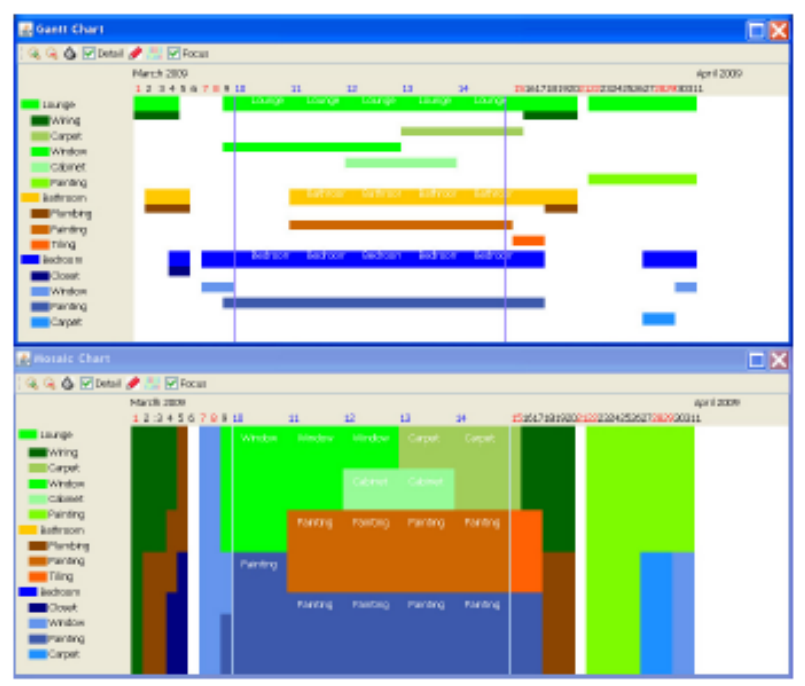

Figure 5: Gantt and mosaic visualizations showing a zoomed-in area.

The main hypothesis investigated was that:

- H1: the focus and context mechanisms improve user performance in terms of speed and accuracy for both mosaic and Gantt visualizations.

More specific hypotheses, related to issues we observed in the visualization styles being compared were:

- H2: the blur mode will be more useful to mosaic users than to Gantt users, and

- H3: the collapsed mode will be more useful to Gantt users than to mosaic users.

The first hypothesis is a statement of the general usefulness of focus and context awareness techniques. While this hypothesis is generally taken for granted in the information visualization literature, we felt that it needed to be explicitly tested in the schedule visualization task. Hypothesis $\mathrm{H} 2$ was based on the fact that mosaics are more visually busy than their Gantt counterparts, specially when the number of sub-tasks increases, and therefore stand to gain more from a technique that highlights areas of interest. As regards $\mathrm{H} 3$, it seemed reasonable to expect that bringing the bars closer together in Gantt would facilitate visualization more than for mosaic, where the shape of the chart does not change. It is known that performance in visual alignment tasks (Vernier acuity) degrades with distance and in the presence of intervening objects [27]. In the Gantt chart, the increase in distance between the target bars and the presence of intervening bars in the expanded mode makes the task considerably more difficult.

\subsection{Methodology}

Each study session started with a tutorial, presented to the participant through a Web page, which described both the Gantt and temporal mosaic visualizations, with a particular emphasis on the focus and context awareness features to be tested. The tutorial consisted of a series of short text segments, each followed by the relevant images, in Gantt and mosaic, of a schedule similar to the one used in the actual experiment task.

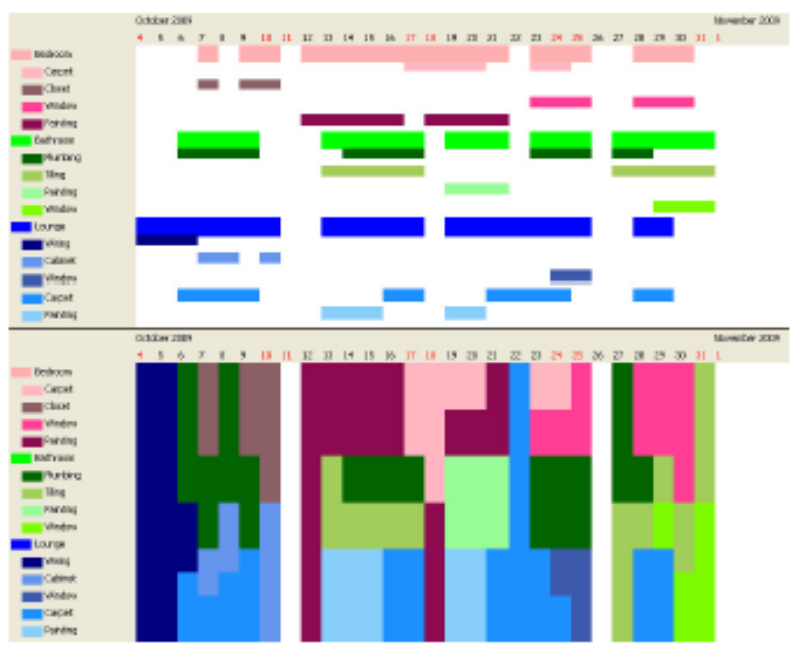

Figure 6: Gantt and mosaic visualizations of the schedule used in the experiment.

At the end of the tutorial the study participants were assigned to one of two sets of task questions which they had to complete. The two sets of questions, which were based on a house renovation project schedule (shown in Figure 6), were identical but the images presented to each participant along with a specific question alternated between one of two different images assigned to that question. For instance, one person answered question 1 while presented with the standard mosaic view of the full schedule, and another person answered the same 
question while presented with the mosaic view of the schedule with the irrelevant parts blurred out. Similar pairings were set up for the other context awareness techniques.

An equal number of participants answered each of the two question sets. Thus, the experiment employed a mixed design which consisted of two major groups with respect to the context awareness features (between subject), and a single block with respect to the underlying type of chart (Gantt or mosaic) presented to the user (within subject). Table 1 shows the list of questions, along with the type of alternate images displayed to the subjects together with each question. The questions required users to assess duration (questions 1, 2, 7, 8, 11 and 12) and concurrency of tasks (questions 3, 4, 5, 6, 9 and 10). The types of images used in the trials are abbreviated as follows:

$\mathrm{MB}$ and GB: Mosaic and Gantt Blurred (blurring of non-focal tasks), MS and GS: Mosaic and Gantt Sharp (no blurring), ME and GE: Mosaic and Gantt Expanded, MC and GC: Mosaic and Gantt Collapsed, MF and GF: Mosaic and Gantt Focused (bifocal lens on), MN and GN: Mosaic and Gantt Normal (bifocal lens off)

A Java application was developed for this experiment and used to present the 12 questions randomly, and one at a time to the participants 1 .For each question, once the participants selected their response from a list of choices (a drop-down list) the application recorded the time taken to

1 The tutorial and experiment question tasks can be accessed on-line at http://ronaldo.cs.tcd.ie/ /uzs/ chronos/.

Table 1: List of task questions by group.

\begin{tabular}{|c|c|c|}
\hline Question & Group 1 & Group 2 \\
\hline $\begin{array}{l}\text { 1) How many days does the plumbing of } \\
\text { the bathroom take in total? }\end{array}$ & MB & MS \\
\hline $\begin{array}{l}\text { 2) How many days does the carpeting of } \\
\text { the lounge take in total? }\end{array}$ & GS & GB \\
\hline $\begin{array}{l}\text { 3) No more than two tasks can take } \\
\text { place concurrently (at the same time) in } \\
\text { a single room. Is this a problem for the } \\
\text { bathroom in the current schedule? }\end{array}$ & MS & MB \\
\hline $\begin{array}{l}\text { 4) No more than two tasks can take } \\
\text { place concurrently (at the same time) in } \\
\text { a single room. Is this a problem for the } \\
\text { bedroom in the current schedule? }\end{array}$ & GB & GS \\
\hline $\begin{array}{l}\text { 5) All the painting work is done by the } \\
\text { same person. Is this person free at least } \\
\text { one day between days } 12 \text { and } 21 \text { ? }\end{array}$ & MB & MS \\
\hline $\begin{array}{l}\text { 6) All the carpeting is done by the same } \\
\text { person. Is this person free at least one } \\
\text { day between days } 17 \text { and } 24 \text { ? }\end{array}$ & GS & GB \\
\hline $\begin{array}{l}\text { 7) How many days does the lounge } \\
\text { renovation take in total? }\end{array}$ & ME & MC \\
\hline $\begin{array}{l}\text { 8) How many days does the bathroom } \\
\text { renovation take in total? }\end{array}$ & GC & GE \\
\hline $\begin{array}{l}\text { 9) Putting carpet in a room and painting } \\
\text { that room can't coincide. Is this a prob- } \\
\text { lem for the bedroom schedule? }\end{array}$ & MC & ME \\
\hline $\begin{array}{l}\text { 10) Putting carpet in a room and } \\
\text { painting that room can't coincide. Is this } \\
\text { a problem for the lounge schedule? }\end{array}$ & GE & GC \\
\hline $\begin{array}{l}\text { 11) All the painting work is done by the } \\
\text { same person between days } 12 \text { and } 21 \text {. } \\
\text { How many days will the painter be on } \\
\text { site in total? }\end{array}$ & MN & MF \\
\hline $\begin{array}{l}\text { 12) All the windows are fitted by the } \\
\text { same carpenter between days } 23 \text { and } \\
\text { 31. How many days will the carpenter be } \\
\text { on site in total? }\end{array}$ & GF & GN \\
\hline
\end{tabular}

answer the question, and then asked the participants to rate the difficulty of the question they had just answered using a scale that ranged from 1 to 7 ( 1 being easy and 7 being difficult). The order of presentation of questions was randomized for each experiment in order to avoid learning effects. Only the actual time to answer each question was recorded. The timer stopped immediately after the answer was selected, and the difficulty rating was not timed. The timer only restarted once the difficulty rating was completed and the next question was shown. The tutorial explained that only the time taken to answer each question was recorded, and requested that the questions should be answered as quickly as possible.

In total, 24 subjects took part in this study: 8 females and 16 males. Of this total, 9 were students, 6 academics and 9 had other occupations. The ages of participants ranged from mid-twenties to mid-fifties.

\subsection{Results}

A general summary of results is shown in Figure 7. The bars in all charts are grouped according to question number, so that for any adjacent pair of bars, the bar on the left represents a graph on which a context awareness feature (blur, collapsing, bifocal lens) has been applied. Abbreviations for the different groups described in Table 1 are shown between the bars and question numbers on the horizontal axis.

The top chart shows the mean times taken to answer each question. Analysis of variance revealed a significant main effect for context awareness technique with respect to answer time, but no effect for chart type or significant interactions $[F(9,111)=$ $17.93, p<0.001]$. Across the different awareness conditions, the mean times for Gantt and mosaic charts were quite similar, mosaic users taking about 35.9s to answer a question, and Gantt users taking 33.8s [Paired t-test: $\mathrm{t}(143)=1.23, \mathrm{p}=0.22$, non significant]. However, post-hoc analysis of the context awareness techniques (using paired t-tests) revealed differences mainly when answers were aggregated over the basic visualization types. There were significant time differences between answers based on blurred ( $\mathrm{x}=28.6, \mathrm{~s}=16.6)$ and sharp $(\mathrm{x}=32.9, \mathrm{~s}=17.7)$ charts $[\mathrm{t}(71)=-2.13, \mathrm{p}<0.05]$. Overall, Gantt users seem to have benefited slightly more from the blur-out effect than mosaic users, with a mean speedup of $6 \mathrm{~s}$ (of blur over sharp) for the former, versus $2.7 \mathrm{~s}$ for the latter, though these results are not statistically significant.

Differences were also found consistently $[\mathrm{t}(47)=$ $-2.05, p<0.05$ ] between times for collapsed ( $x$ $=28.7, \mathrm{~s}=12.9$ ) and expanded charts ( $\mathrm{x}=36.8$, $s=23.8)$. This time mosaic users were the main beneficiaries in general, attaining a speedup of $11.6 \mathrm{~s}$ for collapsed over expanded for mosaic [t(16) =1.7,p $=0.09$ ], versus a speedup of 4.7 for Gantt [non sig.]. 

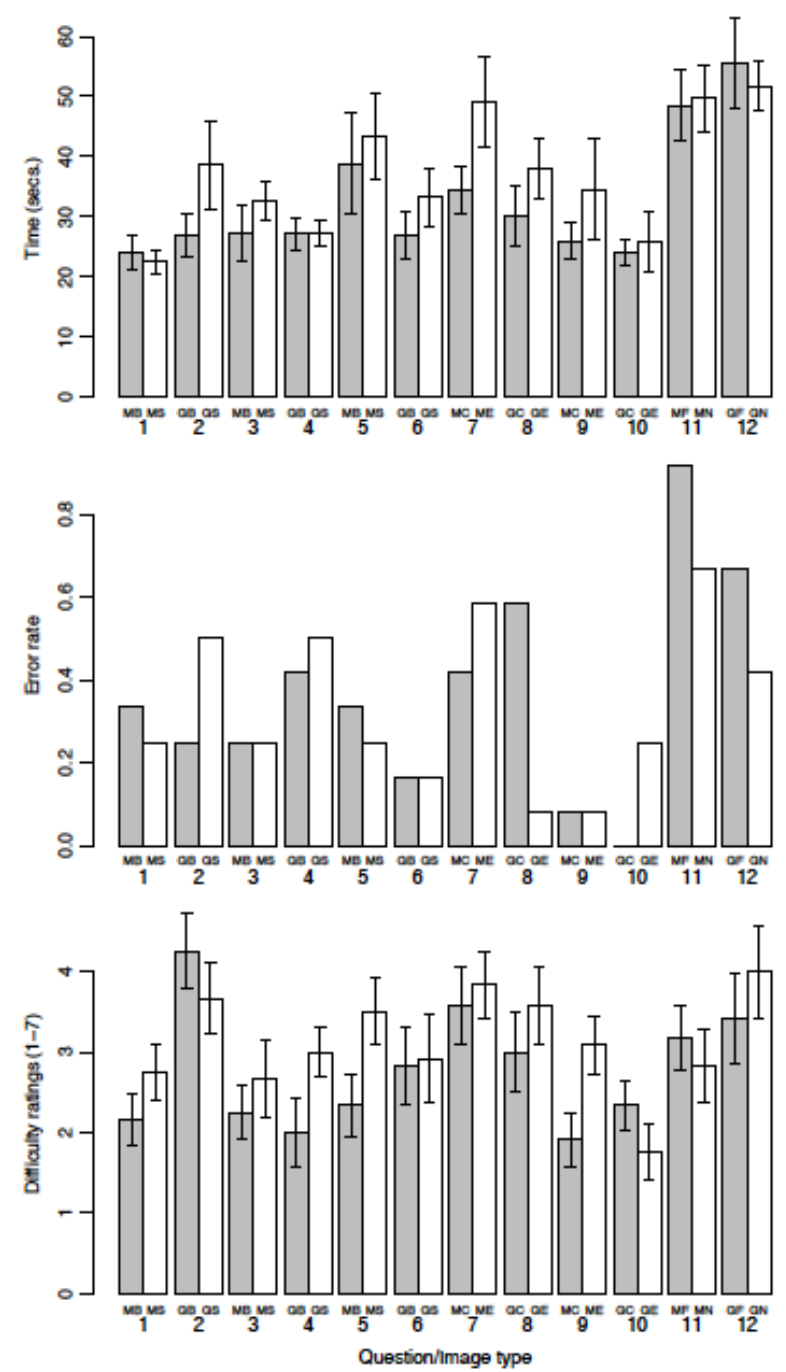

Figure 7: Mean time taken to answer the questions, error rates, and difficulty ratings by question number (see Table 1).

Finally, the bifocal technique presents a mixed picture: mosaic users appear to have benefited from it as far as answer time is concerned but Gantt users seem to have been hindered by the technique. Our results for this condition are, however, inconclusive. When error rates are analysed, it becomes evident that while the diffculties Gantt users found with the bifocal technique are reflected in the higher error rates, the apparent improvement in answer times for mosaic users of the bifocal technique comes at the expense of accuracy. There was a mean increase of $36 \%$ in error for the bifocal lens condition in mosaic and a $60 \%$ increase in error for Gantt [2 (1) $=3.37, p=0.06]$. This is likely to indicate that users misinterpreted the display, since the difficulty ratings for questions 11 and 12 are consistent with their answer times. This conjecture however needs to be investigated further.

Other than these effects, the results for error rates are less clear. This is probably due to the fine level of detail involved in the tasks and the emphasis on timing we expressed in the pre-trial tutorial. Blurring does not appear to have helped with questions involving assessment of duration, but error rates were surprisingly high for mosaic on question 5 , which involved assessing overlaps. On the other hand, collapsing the representation helped the same type of assessment for Gantt charts in question 10. As mentioned above, ratings were generally consistent with performance in that they reflected the times taken to answer each question: questions rated more difficult took longer to answer than questions rated easy. Correlation was observed for both Gantt (Pearson's product-moment $r=0.27$, $p<$ $0.01)$ and mosaic charts $(r=0.26, p<0.01)$.

\subsection{Discussion}

The main hypothesis $(\mathrm{H} 1)$ was generally confirmed though with some caveats, as reported above. Blurring and collapsing yielded clear improvements, whereas the results for the bifocal technique proved inconclusive.

As regards our second hypothesis $(\mathrm{H} 2)$, we had expected that the blur technique would be specially useful in conjunction with the mosaic visualization. The results, however, failed to support or refute this hypothesis. Moreover, as noted above, in question 5 the blurring seems to have mislead the mosaic users, who answered the question more quickly when using the blurred-out version but also made more mistakes. Upon examining the chart that accompanied the question we noticed that one of the colours which was supposed to be highlighted (a pale green) was quite similar to a blurred-out colour. This could have interfered with the users' ability to differentiate between a time interval in which the painter was free and one for which a painting task was scheduled. This emphasizes the need for smooth transitions between sharp and blurred representations to be implemented in order to indicate more clearly to the user which areas are fading out of focus. A possible solution is presented in section 5.1, below.

Surprisingly, hypothesis $\mathrm{H} 3$, that the collapsed mode would facilitate the assessment of concurrency (questions 9, and 10), particularly in Gantt charts, was refuted. Mosaic users benefited the most from this technique. Assessment of duration, in particular, seems to have improved for mosaic (question 7 ) in collapsed mode, though the results are still inconclusive for Gantt charts (question 8). This analysis motivated the design changes described in Section 5.3.

Finally, we conjecture that the high error rates for bifocal lens condition probably stem from difficulties in aligning the event representations (bars and mosaic tiles) to the calendar days at the top of the chart, as the scale changes. Users might have felt tempted to guess duration based on absolute width of the corresponding regions on the charts rather than refer to the distorted time scale. In fact, about 
$69 \%$ of the answers given overestimated the correct duration, which supports this conjecture.

\section{IMPROVING FOCUS AND CONTEXT AWARENESS}

The results of the evaluation demonstrated that further improvements had to be made to our interactive prototype system in terms of its focus and context awareness capabilities. In this section we describe some of these improvements.

\subsection{Control over Blurring}

The initial prototype applied a certain level of blurring to all tasks or sub-tasks that were not selected to visually separate them from what was selected by the user. However, depending on the actual activity being performed by the users it may be necessary to increase or decrease the contrast between selected and unselected tasks. For instance when it is necessary to have more context awareness (e.g. see a sub-task along with other tasks in its neighbourhood) it would be useful to decrease the blurring. On the other hand, when the user wants to for example measure the length of a task without worrying about other tasks, these other tasks could be blurred out completely. Our latest prototype gives the user control over blurring level through a slider which can be interactively manipulated. Figure 8 shows two different levels of blurring applied by the user for the same selected sub-task.

\subsection{Transition between Viewing of Tasks and Sub-tasks}

The latest prototype also provides visual control over the level of collapsing or expanding sub-tasks in mosaic visualization. Using this tool users can visually move between viewing tasks and their subtasks by moving a slider which changes the colour associated with a task to the colour of its individual sub-tasks. Figure 9 shows two different positions on this transition slider and its effect on the resulting mosaic visualization.

It is interesting to note that this type of transition slider is not needed in the Gantt visualization, as our initial implementation of Gantt always shows the task time lines even when their sub-tasks are expanded.

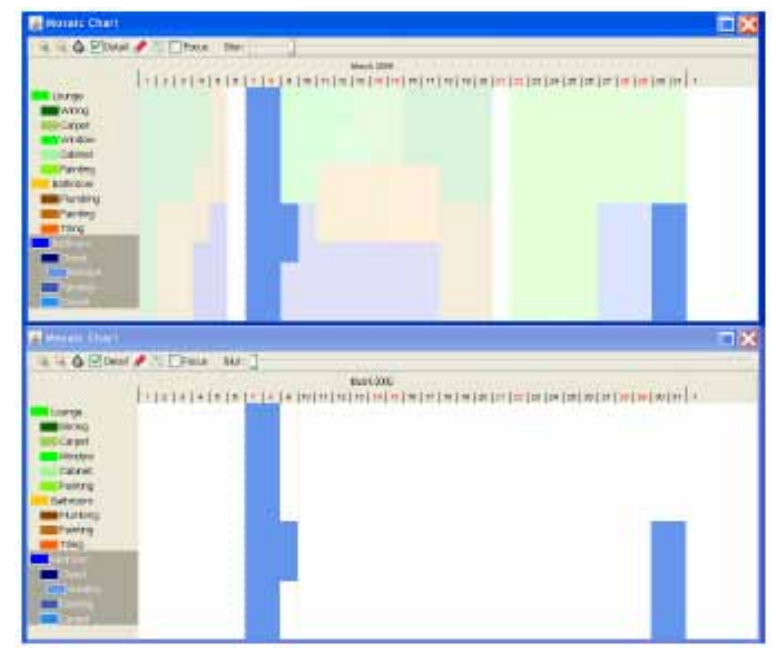

Figure 8:Control over the blurring level in mosaic visualization.

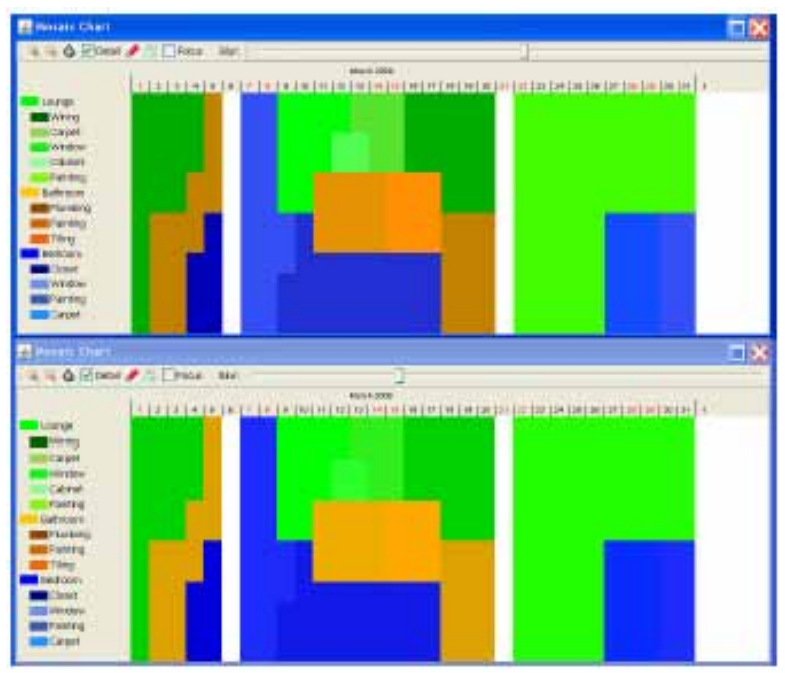

Figure 9: Transition between collapsed and expanded sub-tasks in mosaic visualization.

\subsection{Combining Gantt and Mosaic}

Our various user evaluations comparing Gantt and mosaic visualizations have shown that each visualization has its own strong points in terms of providing for focus and context awareness. It is therefore natural to attempt to somehow combine the two visualizations with the aim of improving their capabilities. Our latest prototype implements two different techniques for combining Gantt and mosaic time lines.

The first technique, as shown in Figure 10, allows the user to simply display Gantt time lines over mosaic by holding down the SHIFT key while in mosaic visualization mode. The benefit of this option is to provide a means of quickly seeing the length of a task, particularly when the mosaic visualization has not been able to draw the task area continuously (for details on this problem see [17]).

The prototype, however, does not implement a means of displaying mosaic over Gantt time lines. 
The reason for this is that the mosaic visualization uses the chart area fully (except for when there are no tasks scheduled), and therefore displaying mosaic over Gantt would cover the Gantt chart completely. Although it would be possible to increase the transparency of the mosaic overlay, this would result in a combination similar to the one already achieved by displaying Gantt over mosaic.

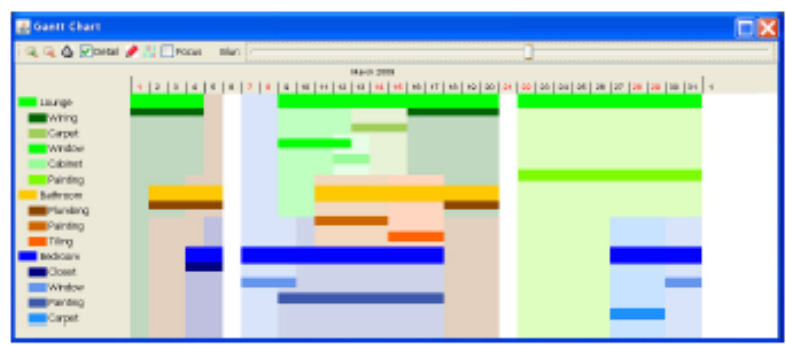

Figure 10: Gantt chart over mosaic visualization.

Our second technique, on the other hand, combines Gantt and mosaic visualizations at a sub-task level. Figure 11 shows an example of this combination, which allows expanding the Gantt task time lines by displaying the sub-tasks in mosaic visualization. The main benefit of this combined visualization is to allow easier viewing of a specific task and its subtasks in terms of their time duration, co-occurrence, etc at a local level than what is possible with Gantt or mosaic visualizations alone. In addition, as the number of sub-tasks of a task would be far smaller than all the sub-tasks in a project schedule, the mosaic visualization becomes much easier to draw and interpret at the individual task level.

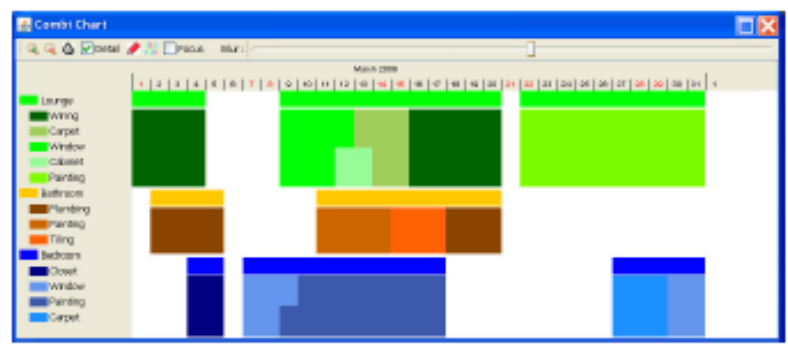

Figure 11: Gantt chart with mosaic sub-tasks visualization.

\section{CONCLUSIONS AND FURTHER WORK}

In this paper we presented an evaluation of a comprehensive range of techniques designed to improve the focus and context awareness capabilities of conventional Gantt charts, as well as our own space-saving mosaic time line visualization. Although the user study has shown the benefits of at least two of these visualization techniques, namely blurring and collapsing of task hierarchies, it has also allowed us to identify further improvements that could be made to the prototype system in order to address the shortcomings we identified. We have now implemented a number of these improvements, along with the additional functionality which have also been discussed in this paper.

We are currently in the process of investigating possible applications of our mosaic visualization, and its focus and context awareness capabilities, in areas other than project scheduling. One particular area of interest is the visualization of inter-related attribute sets, for instance in searching and finding real-estate, in a manner similar to what is done by the Attribute Explorer [24], by allowing interactive comparison of their various attributes such as prices, number of bedrooms, etc.

\section{Acknowledgments}

The first author's research was partially funded by Science Foundation Ireland under the Research Frontiers program.

\section{REFERENCES}

[1] W. Aigner, S. Miksch, W. M Aijller, H. Schumann, and $C$. Tominski. Visualizing time-oriented data: A systematic view. Computers \& Graphics, 31(3):401409, 2007.

[2] W. Aigner, S. Miksch, B. Thurnher, and S. Biffl. Planning lines: novel glyphs for representing temporal uncertainties and their evaluation. In Proceedings 9th International Conference on Information Visualisation, pages 457-463. IEEE Press, July 2005.

[3] O. Alonso, M. Gertz, and R. Baeza-Yates. Search results using timeline visualizations. In SIGIR '07: Procs. of the 30th annual ACM SIGIR Conference on Research and Development in Information Retrieval, pages 908-908, New York, 2007. ACM Press.

[4] P. Andre, M. L. Wilson, A. Russell, D. A. Smith, A. Owens, and m.c. schraefel. Continuum: designing timelines for hierarchies, relationships and scale. In UIST '07: Proceedings of the 20th annual ACM symposium on User interface software and technology, pages 101-110, New York, NY, USA, 2007. ACM.

[5] M. Apperley, D. Fletcher, B. Rogers, and

$\mathrm{K}$. Thomson. Interactive visualisation of a travel itinerary. In AVI '00: Proceedings of the working conference on Advanced visual interfaces, pages 221-226, New York, NY, USA, 2000. ACM.

[6] B. B. Bederson, A. Clamage, M. P. Czerwinski, and G. G. Robertson. DateLens: A fisheye calendar interface for PDAs. ACM Transactions on ComputerHuman Interaction, 11(1):90-119, 2004.

[7] M. Burch, F. Beck, and S. Diehl. Timeline trees: visualizing sequences of transactions in information hierarchies. In AVI '08: Proceedings of the working conference on Advanced visual interfaces, pages 75-82, New York, NY, USA, 2008. ACM. 
[8] S. Card, B. Sun, B. Pendleton, J. Heer, and J. Bodnar. Timetree: Exploring time changing hierarchies. In IEEE Symposium On Visual Analytics Science And Technology, pages 3-10, 2006.

[9] R. Dachselt and M. Weiland. TimeZoom:

a flexible detail and context timeline. In $\mathrm{CHI}$ '06: Extended Abstracts on Human Factors in Computing Systems, pages 682-687, New York, 2006. ACM Press.

[10] G. W. Furnas. Generalized fisheye views. In $\mathrm{CHI}$ '86: Proceedings of the SIGCHI conference on Human factors in computing systems, pages 16-23, New York, NY, USA, 1986. ACM Press.

[11] S. Havre, E. G. Hetzler, P. Whitney, and L. T. Nowell. Themeriver: Visualizing thematic changes in large document collections. IEEE Transactions on Visualization and Computer Graphics,8(1):9-20, 2002.

[12] D. Huynh. SIMILE-Timeline. http://simile.mit. edu/timeline/, 2008.

[13] D. Huynh, S. Drucker, P. Baudisch, and C. Wong. Time quilt: scaling up zoomable photo browsers for large, unstructured photo collections. In $\mathrm{CHI}$ '05: Conference on Human Factors in Computing Systems, pages 1937-1940. ACM New York, NY, USA, 2005.

[14] G. M. Karam. Visualization using timelines. In Procs of the SIGSOFT international symposium on Software Testing and Analysis,pages 125-137. ACM,1994.

[15] R. Kosara, S. Miksch, and H. Hauser. Focus+context taken literally. IEEE Computer Graphics and Applications,22(1):22-29, Jan/Feb 2002.

[16] Y. K. Leung and M. D. Apperley. A review and taxonomy of distortion-oriented presentation techniques. ACM Trans. Comput.-Hum. Interact., 1(2):126-160, 1994.

[17] S. Luz and M. Masoodian. Visualisation of parallel data streams with temporal mosaics. In Procs. of the 11th Intl. Conference on Information Visualisation, pages 196-202, Zurich, 2007. IEEE Press.

[18] S. Luz, M. Masoodian, D. McKenzie, and W. V. Broeck. Chronos: A tool for interactive scheduling and visualisation of task hierarchies. In 13th International Conference on Information Visualisation, IV09, pages 241-246. IEEE Press, July 2009.

[19] J. D. Mackinlay, G. G. Robertson, and S. K. Card. The perspective wall: detail and context smoothly integrated. In $\mathrm{CHI}$ '91: Proceedings of the SIGCHI conference on Human factors in computing systems, pages 173-176, New York, NY, USA, 1991. ACM.

[20] M. Masoodian, D. Budd, and B. Rogers. A comparison of linear and calendar travel itinerary visualizations for personal digital assistants. In OZCHI'04: Proceedings of the CHISIG Annual Conference on Human-Computer Interaction, pages 124-133. Ergonomics Society of Australia, 2004.
[21] C. Plaisant, B. Milash, A. Rose, S. Widoff, and B. Shneiderman. LifeLines: visualizing personal histories. In CHI '96: Proceedings of the SIGCHI conference on Human factors in computing systems, pages 221-227, New York, 1996. ACM Press.

[22] S. F. Silva and T. Catarci. Visualization of linear time-oriented data: A survey. In Proceedings of the First International Conference on Web Information Systems Engineering (WISE), volume 1, pages 310319, Hong Kong, 2000. IEEE Computer Society.

[23] R. Spence. Information visualization.AddisonWesley Harlow, 2nd edition, 2007.

[24] R. Spence and L. Tweedie. The attribute explorer: information synthesis via exploration. Interacting with Computers, 11(2):137-146, 1998.

[25] E. R. Tufte. Project management graphics: ET notebooks (Ask ET. http://www.edwardtufte. com/ bboard/q-and-a-fetch-msg?msg _id=000076. (retrieved March 2010).

[26] E. R. Tufte. The Visual Display of Quantitative Information.Graphics Press, 2nd edition, 2001.

[27] S. J. Waugh and D. M. Levi. Spatial alignment across gaps: contributions of orientation and spatial scale. Journal of the Optical Society of America A: Optics, Image Science, and Vision, 12(10):2305-2317, 1995. 